\title{
a-SiC:H Based Devices as Optical Demultiplexers
}

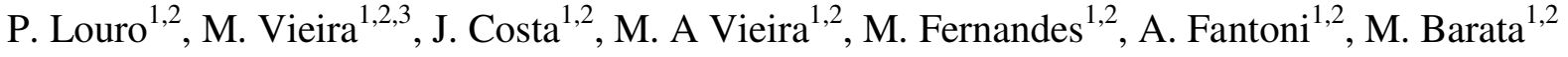 \\ ${ }^{1}$ Electronics Telecommunications and Computer Dept, ISEL, Lisbon, Portugal \\ ${ }^{2}$ CTS-UNINOVA, Lisbon, Portugal. \\ ${ }^{3}$ DEE-FCT-UNL, Quinta da Torre, Monte da Caparica, 2829-516, Caparica, Portugal
}

\begin{abstract}
In this paper we present results on the use of a multilayered a-SiC:H heterostructure as a wavelength-division demultiplexing device (WDM) for the visible light spectrum. The WDM device is a glass/ITO/a-SiC:H (p-i-n)/ a-SiC:H(-p)/Si:H(-i)/SiC:H (-n)/ITO heterostructure in which the generated photocurrent at different values of the applied bias can be assigned to the different optical signals.

The device was characterized through spectral response measurements, under different electrical bias. Demonstration of the device functionality for WDM applications was done with three different input channels covering wavelengths within the visible range. The recovery of the input channels is explained using the photocurrent spectral dependence on the applied voltage. The influence of the optical power density was also analysed.

An electrical model, supported by a numerical simulation explains the device operation. Short range optical communications constitute the major application field, however other applications are also foreseen.
\end{abstract}

\section{INTRODUCTION}

In optical communications one of the major issues of data transmission is the bandwidth of the channel, i.e. the capacity and speed of the transmission [1]. Wavelength division demultiplexing (WDM) is a common technique used to increase the bandwidth of the system. It consists on injecting different optical signals associated to different wavelengths into the same physical transmission medium, the optical fiber. The multiplexed signals travel along the fiber and are recovered at the reception end using a demultiplexer device. Several devices such as diffraction gratings, dielectric films, array waveguide gratings, fiber Bragg gratings, are used for the implementation of the WDM technique.

In this paper we discuss the use of a multilayered a-SiC:H structure as a WDM device in the visible light spectrum [2], which is the transmission window used in many short range optical communication systems [3]. These multilayered structures work as wavelength separation devices [4] with a spectral sensitivity tuned by the external applied voltage.

\section{EXPERIMENTAL DETAILS}

\section{Device Architecture}

In Figure $1 \mathrm{a}$ ) it is shown the configuration of the WDM device, which is composed by two stacked p-i-n photodiodes: pin1 and pin2. The front photodiode (pin1) based on a-SiC:H has a thin absorber layer $(200 \mathrm{~nm})$ which holds the collection of short wavelengths. 
The back one (pin2) is based on a$\mathrm{Si}: \mathrm{H}$ and its absorber is thicker $(1000 \mathrm{~nm})$ which enhances the collection of long wavelengths. The design of each p-i-n photodiode of the device provides two sensitive regions, both optimized for the collection of short and long visible wavelengths. This feature which is the key for the wavelength optical signal separation of the WDM device is illustrated in Figure 1b) that shows the profile of the generation rate along the semiconductor for three different wavelengths covering the short $(470 \mathrm{~nm})$ the medium $(550 \mathrm{~nm})$ and the long $(650$ $\mathrm{nm}$ ) wavelengths of the visible spectrum. The multilayered semiconductor structure is deposited by PE-CDV on a glass substrate between two electrical contacts of transparent conductive oxide (TCO) produced by sputtering. Deposition conditions are described elsewhere [5].

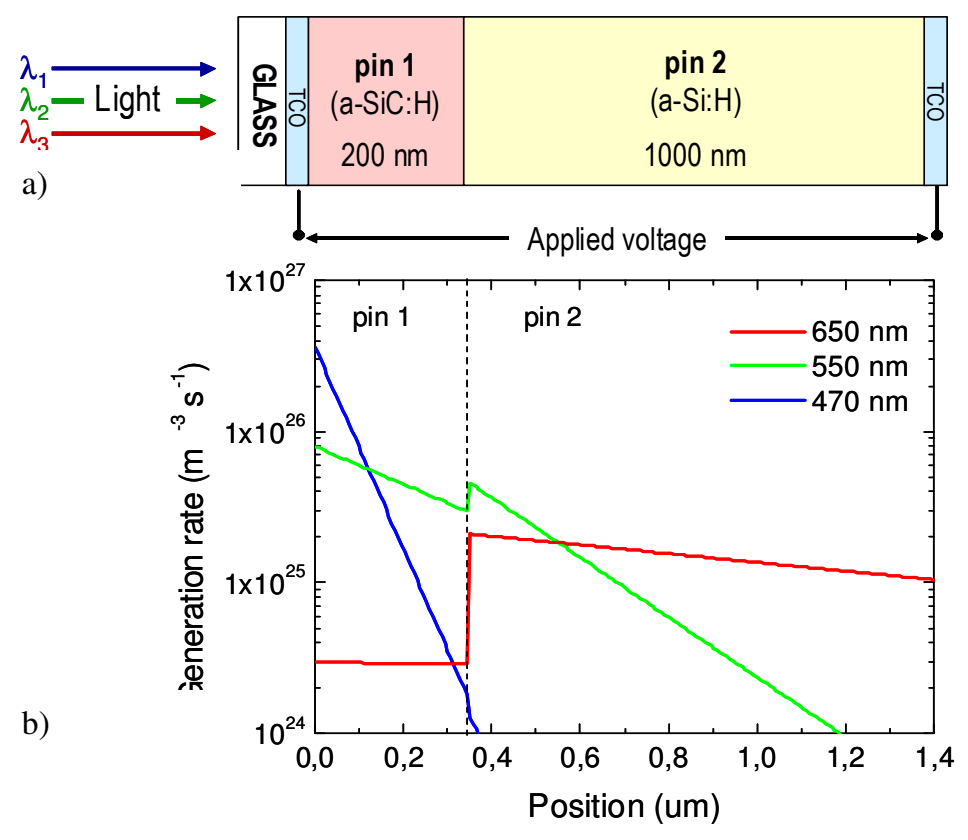

Figure 1. a) Schematic cross section of the WDM device; b) Generation rate profile of the WDM device for three wavelengths $(470 \mathrm{~nm}, 550 \mathrm{~nm}, 650 \mathrm{~nm})$.

\section{RESULTS AND DISCUSSION}

\section{A. Wavelength sensitive device}

Figure 2 shows the spectral photocurrent of the WDM device measured within the visible spectrum (400-800 nm) under forward and reverse bias (- 10V up to +3V) applied between both TCO electrodes.

Results show that in the long wavelength range $(>600 \mathrm{~nm})$ the spectral response is independent of the applied bias. An opposite behavior is found in the short wavelength range as, in this part of the spectrum, the collection strongly increases with the reverse bias.

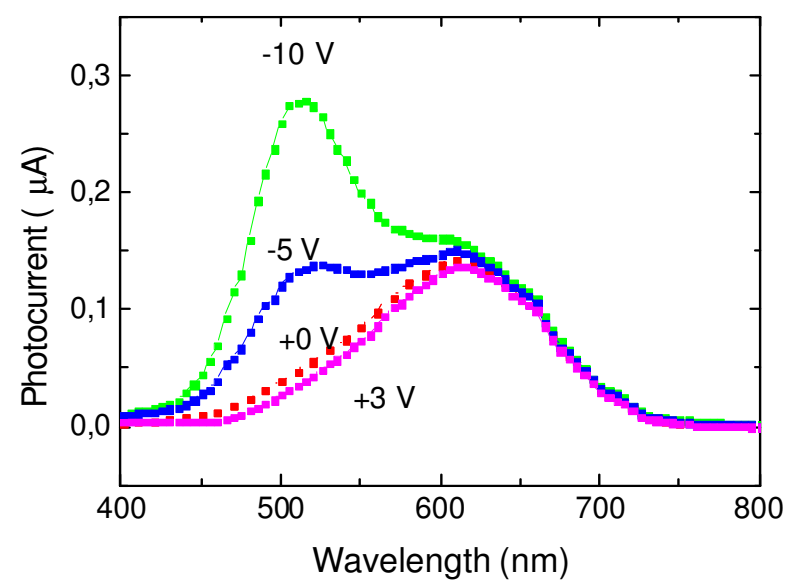

Figure 2.Spectral response of the WDM device at different applied bias. 


\section{B. Wavelength division multiplexing device}

In Figure 3 it is displayed the photocurrent signal obtained with the WDM device under single and combined modulated light bias of long, medium and short wavelengths: Red (R: $626 \mathrm{~nm})$, Green (G: $524 \mathrm{~nm}$ ) and Blue (B: 470nm) illuminating the device from the glass side (see Figure 1).

The generated photocurrent is measured under negative (-8V; solid arrow) and positive $(+1 \mathrm{~V}$, dot arrow $)$ bias. The light modulation frequency of each channel was chosen to be multiple of the others to ensure a synchronous relation of ON-OFF states along each cycle. For each independent wavelength, the output optical powers were adjusted to give different signal magnitudes at $-8 \mathrm{~V}$ (solid arrows). The correspondent photocurrent signals at $+1 \mathrm{~V}$ are also displayed (dotted arrows). The reference level was assumed when all the input channels were OFF.

Data show that under single long wavelength modulated light $(624 \mathrm{~nm})$ the magnitude of the photocurrent signal for forward and reverse bias, is similar, which suggests a weak dependence on the applied voltage, as already indicated by the spectral data of Figure 2. For shorter $(470 \mathrm{~nm})$ and medium $(524 \mathrm{~nm})$ wavelengths the behavior is quite different,

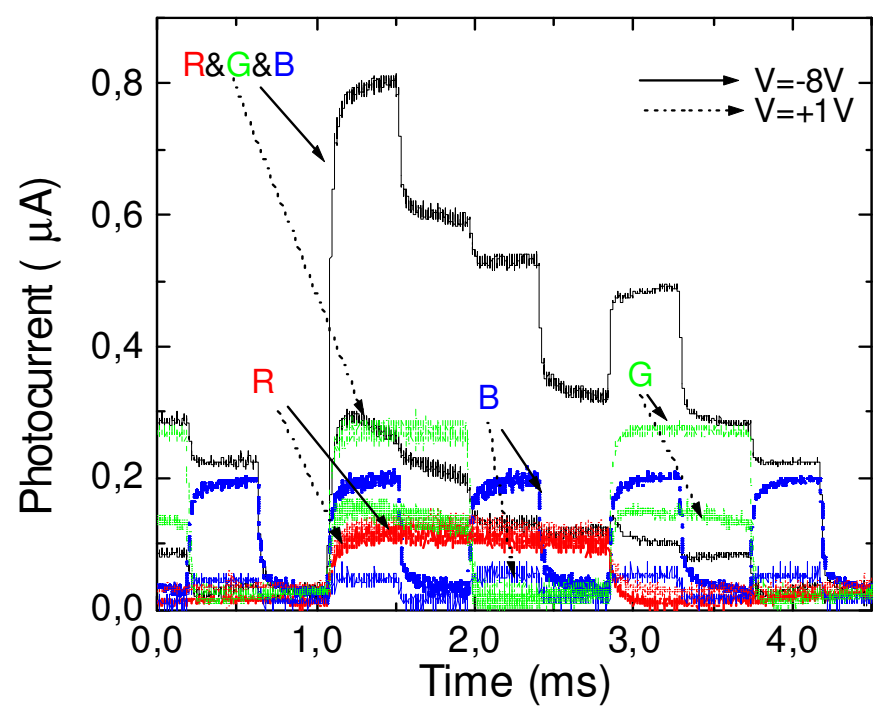

Figure 3. Multiplexed signals obtained under reverse (solid arrow) and forward (dotted arrow) bias using single (R, G and B) and combined (R\&G\&B) optical bias of different wavelengths (R: $626 \mathrm{~nm}, \mathrm{G}: 524 \mathrm{~nm}$ and B: 470nm) as the signal exhibits,respectively, a strong and medium decrease under forward bias.

The multiplexed signal obtained with the combination of the three optical sources exhibits, under reverse bias, eight different levels that correspond each to different optical bias states. As the electrical bias goes from reverse to forward the magnitude decreases and the levels of the threshold photocurrent associated to each optical state become closer and less defined, which is in agreement with the results displayed in Figure 2 that showed the extinction of the photocurrent caused by optical signals of short wavelengths. This mechanism can be used for the identification of the input channels using the photocurrent signal obtained under forward and reverse signals and comparing the magnitude of the variations in each optical state.

Under reverse bias, the sum of the input channels is lower than the correspondent multiplexed signals. This optical amplification, mainly on the ON-ON states, suggests capacitive charging currents due to the time-varying nature of the incident lights. Under forward bias the device becomes blind to the front photodiode (Figure 1 and 2) and the blue component of the combined spectra falls into the dark level, allowing the recognition of the red and green input channels. By comparing the signals under forward and reverse bias and using a simple algorithm that takes into account the different sub-level behaviors it is possible to decode the RGB input signals.

To recover the transmitted information ( 8 bit per wavelength channel) the multiplexed signal, during a complete cycle $(0<\mathrm{t}<\mathrm{T})$, was divided into eight time slots, each corresponding to one bit where the independent optical signals can be ON (1) or OFF (0). As under forward bias the WDM device has no sensitivity to the blue channel (Figure 3), the red and green transmitted information can be identified from the multiplexed signal at $+1 \mathrm{~V}$. The highest level corresponds to both channels ON ( $R \& G: R=1, G=1)$, and the lowest to the OFF-OFF stage $(R=0 ; G=0)$. The two levels in-between are related with the presence of only one channel $\mathrm{ON}$, the red $(\mathrm{R}=1, \mathrm{G}=0)$ or the green $(\mathrm{R}=0, \mathrm{G}=1)$. To 
distinguish between these two situations and to decode the blue channel, the correspondent sub-levels, under reverse bias, have to be analyzed. From Figure 3 it is observed that the green channel is more sensitive to changes on the applied voltage than the red, and that the blue only appears under reverse bias. So, the highest increase at $-8 \mathrm{~V}$ corresponds to the blue channel $\mathrm{ON}(\mathrm{B}=1)$, the lowest to the $\mathrm{ON}$ stage of the red channel $(R=1)$ and the intermediate one to the $\mathrm{ON}$ stage of the green $(\mathrm{G}=1)$. Using this simple algorithm the independent red, green and blue bit sequences can be decoded as: R[00001111], $\mathrm{G}[00110011]$ and B[01010010], which is in agreement with the pulse light emitted by each optical channel.

\section{Influence of optical signal intensity}

The identification of the different input channels requires a previous calibration of the transmission signal in order to know the response of the WDM device to each individual channel as the signal attenuation along the transmission medium causes a reduction of the optical intensity at the reception end (WDM device). In order to analyze the influence of this effect the multiplexed signal was acquired with input signals of different optical intensities at $-8 \mathrm{~V}$ and $+1 \mathrm{~V}$. Measurements were made with different levels of increasing optical power, up to $140 \mu \mathrm{Wcm}^{-2}$.

In Figure 4 it is displayed the output photocurrent density variation with the optical bias measured for each optical channel (R: $626 \mathrm{~nm}, \mathrm{G}: 524 \mathrm{~nm}$ and B: $470 \mathrm{~nm}$ ) at $-8 \mathrm{~V}$ and $+1 \mathrm{~V}$.

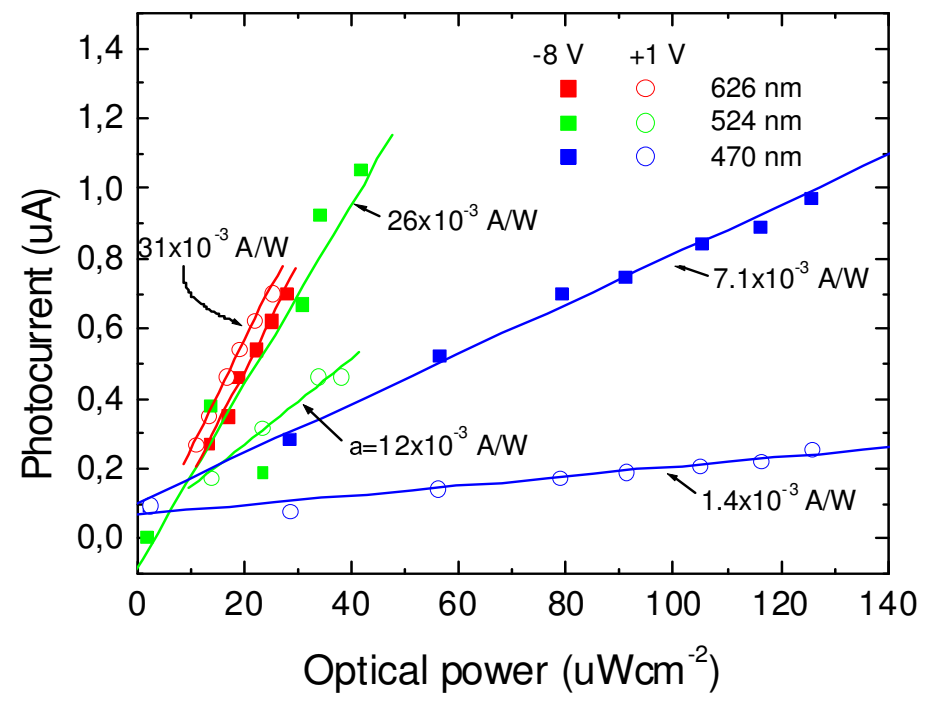

Figure 4 .Photocurrent density variation with the optical bias measured for each optical channel (R: $626 \mathrm{~nm}$, G: $524 \mathrm{~nm}$ and B: $470 \mathrm{~nm}$ ) at $-8 \mathrm{~V}$ (solid symbols) and + 1V (open symbols). The solid lines correspond to linear fits of the experimental data. Slopes $(\square)$ of each plot are also displayed.

Results show that under red illumination the multiplexed signal magnitude under the same intensity conditions is independent on the applied bias. Its magnitude increases with the optical power intensity, exhibiting a linear behavior with a growth rate around $31 \times 10^{-3} \mathrm{~A} / \mathrm{W}$. Under green and blue light the dependence of the photocurrent magnitude is strongly dependent on the polarity of the applied bias as already demonstrated before (Figure 2 and 3). It increases with the optical intensity of each channel either for reverse and forward bias and the growth rate depends on the applied voltage, being higher at reverse bias. Under blue light $(470 \mathrm{~nm})$ the growth rate is 5 times higher at reverse than under forward bias, while under green illumination $(524 \mathrm{~nm})$ this ratio is only of a factor of 2 . This is due to the strong reduction of the device sensitivity for the shorter wavelengths under forward bias (Figures 2 and 3). 


\section{THEORETICAL MODEL}

Based on the experimental results (Figure 3) and device configuration (Figure 1) an electrical model was developed [6], the $a c$ equivalent electrical circuit is displayed in Figure 5a. Two pulsed current sources with different frequencies, $I_{1}$ and $I_{2}$, are used to simulate the input blue and red channels, respectively. The green channel is simulated by two sources with the same frequencies, $\mathrm{I}_{3}$ and $\mathrm{I}_{4}$, since the green absorption occurs across both front and back intrinsic layers. Capacitors, $\mathrm{C}_{1}$ and $\mathrm{C}_{2}$, and resistors, $R_{1}$ and $R_{2}$ are used to model, respectively, the transient capacitance due to the minority carriers trapped in both p-i-n junctions and the dynamic resistances under different $d c$ biasing conditions. If the device is biased negatively, Q1 and Q2 are operating both in the active-reverse regions. The p-n internal junction is forward-biased (lower $\mathrm{R}_{1}$ ) and the external voltage drops across both front and back reverse-biased junctions. Under positive bias the internal junction becomes always reverse-biased (higher $\mathrm{R}_{1}$ ).

The Kirchhoff's laws for the simplified ac equivalent circuit give the time periodic linearized state equation (see equations 1 ).

$$
\frac{d v_{1,2}}{d t}=\left[\begin{array}{cc}
-\frac{1}{R_{1} C_{1}} & \frac{1}{R_{1} C_{1}} \\
\frac{1}{R_{1} C_{2}} & -\frac{1}{R_{1} C_{2}}-\frac{1}{R_{2} C_{2}}
\end{array}\right] v_{1,2}(t)+\left[\begin{array}{c}
\frac{1}{C_{1}} \\
\frac{1}{C_{2}}
\end{array}\right] i_{1,2}(t), \quad i(t)=\left[\begin{array}{ll}
0 & \frac{1}{R_{2}}
\end{array}\right] v_{1,2}(t)
$$

with $v_{l}(t)$ and $v_{2}(t)$ the emitter-base voltages of Q1 and Q2 transistors and $i(t)$ the generated photocurrent under transient conditions. We have used MATLAB as a programming environment and the four order Runge-Kutta method to solve the state equations. Figure $5 \mathrm{~b}$ displays the current, $i(t)$, under positive (open symbols) and negative (solid symbols) $d c$ bias. The input current sources $\left(\mathrm{I}_{1}, \mathrm{I}_{2}, \mathrm{I}_{3}\right.$ and $\mathrm{I}_{4}$ ) are also displayed (dash lines). We have used as input parameters the experimental values (Figure 3). To validate the model the experimental multiplexed signal at $-8 \mathrm{~V}$ and $+1 \mathrm{~V}$ is also shown (lines).

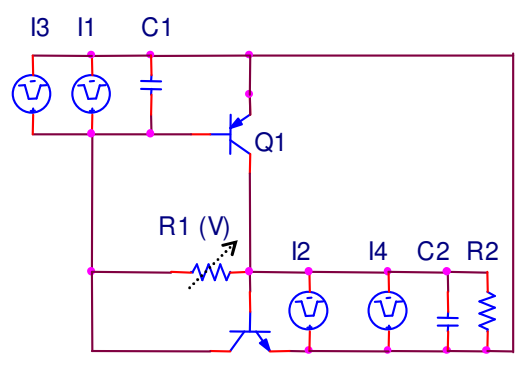

Q2

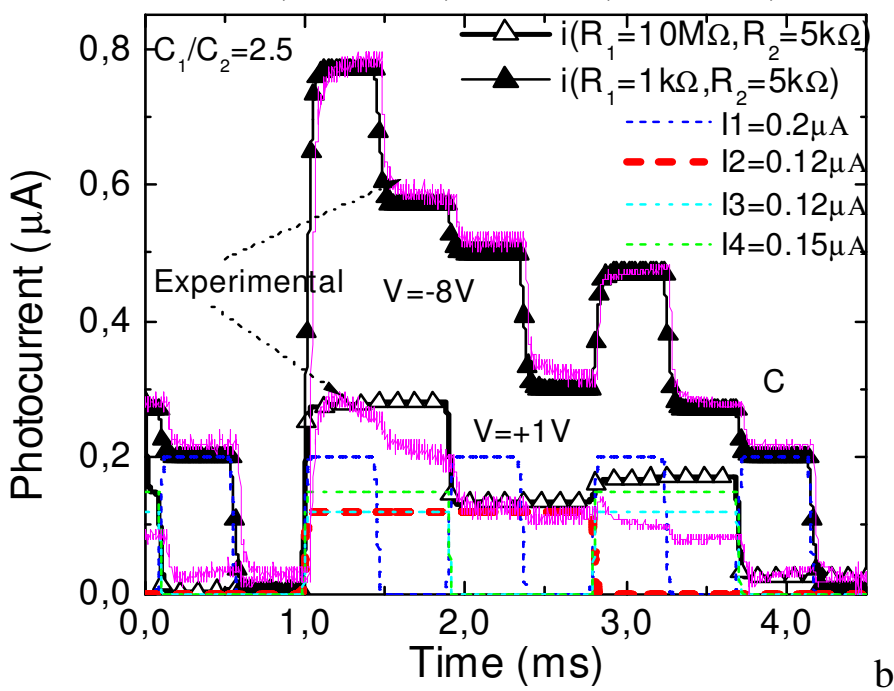

b)

Figure 5 a) Simplified ac equivalent electrical circuit. b) Multiplexed simulated (symbols) and experimental (solid lines) results under positive $\left(\mathrm{R}_{1}=10 \mathrm{M} \Omega ;+1 \mathrm{~V}\right)$ and negative $\left(\mathrm{R}_{1}=1 \mathrm{k} \Omega ;-8 \mathrm{~V}\right) d c$ bias. The current sources used as input channels (dash lines) are displayed. 
Good agreement between experimental and simulated data was observed. Under transient conditions the circuit becomes a bucket-brigade device. The device is a transmission system able to store and transport the minority carriers generated by the current pulses, through capacitors $\mathrm{C}_{1}$ and $\mathrm{C}_{2}$. Under negative bias, once the blue channel is $\mathrm{ON}$, its emitter-base becomes optically forward biased and $\mathrm{C}_{2}$ is rapidly charged in inverse polarity of $\mathrm{C}_{1}\left(i_{C 1}(t) \times C_{2}=-i_{C 2}(t) \times C_{1}\right)$ with an input voltage in which a $d c$ value is inserted for clamping, resulting in a reinforcement of the reverse bias at $\mathrm{Q} 2$. The current source keeps filling the capacitors during the pulse and the transferred charge between $C_{1}$ and $C_{2}$ reaches the output terminal as a capacitive charging current.

The presence of the red channel changes, in the opposite way, the charge of both capacitors.If the green channel is $\mathrm{ON}$ the current is the balance between $\mathrm{I}_{3}$ and $\mathrm{I}_{4}$ contributions (Figure 2 and Figure $3)$. With all the input channels $\mathrm{ON}$, the packets of charge stored at $\mathrm{C}_{1}\left(\mathrm{I}_{1}, \mathrm{I}_{3}\right)$ are sequentially transferred to $\mathrm{C}_{2}$ and together with the minority carriers generated at the base of $\mathrm{Q} 2\left(\mathrm{I}_{2}, \mathrm{I}_{4}\right)$ flow across the circuit. If a small positive voltage is applied the resistance, $\mathrm{R}_{1}$ is enlarged and the carriers collected come only from the input channels at $\mathrm{Q} 2\left(\mathrm{I}_{2}, \mathrm{I}_{4}\right)$, allowing the demultiplexing of the previous multiplexed signal (Figure 3) by switching between positive and negative voltages.

\section{ONCLUSIONS}

In this paper we present results on the use of a multilayered a-SiC:H heterostructure as a wavelength-division demultiplexing device for the visible light spectrum. It was demonstrated, for three different input channels, the device functionality for WDM applications. A recognition algorithm for the reconstruction of the input channels was proposed and tested under different conditions of optical intensity. The proposed electrical model of the WDM device and the solution of the respective circuit equations show an excellent agreement with the experimental data. More work has to be done to confirm the reliability of the device in the correct recognition of random input sequences.

\section{ACKNOWLEDGEMENTS}

This work supported by Fundação Calouste Gulbenkian and by POCTI/FIS/70843/2006.

\section{REFERENCES}

1. Michael Bas, Fiber Optics Handbook, Fiber, Devices and Systems for Optical Communication, Chap, 13, Mc Graw-Hill, Inc. 2002.

2. P. Louro, M. Vieira, M. A. Vieira, M. Fernandes, A. Fantoni, G. Lavareda, C.N. Carvalho, Amorphous and Polycrystalline Thin-Film Silicon Science and Technology — 2009, MRS Proceedings Volume 1153.

3. P. Louro, M. Vieira, M A Vieira, M. Fernandes, A. Fantoni, C. Francisco, M. Barata, Physica E 41 (2009) 1082-1085.

4. M. Topic, H. Stiebig, M. Krause and H. Wagner, Appl. Phys. Lett. 78 (2001), p. 2387

5. M. Vieira, A. Fantoni, M. Fernandes, P. Louro, G. Lavareda and C.N. Carvalho, Thin Solid Films, 515, Issue 19, 2007, 7566-7570.

6. M. A. Vieira, M. Vieira, M. Fernandes, A. Fantoni, P. Louro, M. Barata, Amorphous and Polycrystalline Thin-Film Silicon Science and Technology - 2009, MRS Proceedings Volume 1153, A08-03 